\author{
Yolanda Martín-Biosca ${ }^{2}$ \\ Carmen García-Ruiz ${ }^{1}$ \\ Maria L. Marina ${ }^{1}$ \\ ${ }^{1}$ Departamento de Química \\ Analítica, Facultad de \\ Química, Universidad de \\ Alcalá, Alcalá de Henares \\ (Madrid), Spain \\ 2Departamento de Química \\ Analítica, Facultad de \\ Farmacia, Universitat de \\ València, \\ Burjassot (Valencia), Spain
}

\section{Fast enantiomeric separation of uniconazole and diniconazole by electrokinetic chromatography using an anionic cyclodextrin: Application to the determination of analyte-selector apparent binding constants for enantiomers}

The enantiomeric resolution of the fungicides uniconazole and diniconazole was performed using electrokinetic chromatography with cyclodextrins as pseudostationary phase (CD-EKC). A systematic evaluation of several chiral selectors was made. The anionic derivative carboxymethylated- $\gamma$-cyclodextrin $(\mathrm{CM}-\gamma-\mathrm{CD})$ was found to be the most appropriate for the enantioseparation of fungicides among all cyclodextrins tested. The influence of some experimental conditions such as nature and buffer $\mathrm{pH}$, chiral selector concentration, and temperature on the enantiomeric separation of the compounds studied was also investigated. The use of a $50 \mathrm{~mm}$ phosphate buffer (pH 6.5) containing $5 \mathrm{~mm} \mathrm{CM- \gamma -CD}$ and a temperature of $50^{\circ} \mathrm{C}$ enabled the baseline enantioresolution of mixtures of uniconazole and diniconazole in less than $5 \mathrm{~min}$. In addition, apparent binding constants for each enantiomer-CM- $\gamma-C D$ pair at several temperatures, as well as thermodynamic parameters for binding were calculated.

Keywords: Uniconazole / Diniconazole / Electrokinetic chromatography / Carboxymethylated $\gamma$ cyclodextrin / Complex mobility / Inclusion complex binding constant / Enantioseparation / Thermodynamic parameters

EL 4119

\section{Introduction}

Uniconazole and diniconazole have fungicidal and plant growth regulating activities. In both cases, the $R$-enantiomer shows stronger fungicidal activity than the S-enantiomer, whereas the latter has higher plant growth regulating activity. On the other hand, uniconazole has higher plant growth regulating activity than diniconazole but is less active as a fungicide. Consequently, products containing a high proportion of the $R$ - and $S$-enantiomer for diniconazole and uniconazole, respectively, have been developed as a high-activity fungicide and an effective plant growth regulator [1, 2]. Reliable and efficient methods for separating the enantiomers are therefore necessary.

Correspondence: Dr. M. L. Marina, Departamento de Química Analítica, Facultad de Química, Universidad de Alcalá, Ctra. Madrid-Barcelona Km. 33.600, 28871 Alcalá de Henares (Madrid), Spain

E-mail: mluisa.marina@uah.es

Fax: $+34-91-8854971$

Abbreviations: $\mathbf{C M}-\beta-\mathbf{C D}$, carboxymethylated- $\beta$-cyclodextrin;

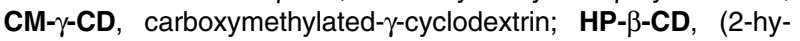
droxy)propyl- $\beta$-cyclodextrin; Succ- $\beta-C D$, succinylated- $\beta$-cyclodextrin; Succ- $\gamma-\mathbf{C D}$, succinylated- $\gamma$-cyclodextrin
Chiral separations are one of the most promising topics in the field of capillary electrophoresis (CE), which has been outlined by several recent review articles [3-8]. In CE, chiral separations can be achieved using various chiral selectors, by means of electrokinetic chromatography (EKC). As chiral selectors, neutral and charged cyclodextrins (CDs) [4-7, 9], bile salts [5], monomeric and polymeric chiral surfactants [10], crown ethers [7], calixarenes [9], proteins [11], macrocyclic antibiotics [12], polysaccharides [9], or ergot alkaloids [9] have been employed. The chiral separation principle relies on the different partition of enantiomers between the bulk solution and the chiral pseudophase.

Inclusion into the cavity of CDs represents the most frequently used approach for chiral separation by CE. Not only neutral CD derivatives, but also charged CDs have been developed. The chiral recognition mechanism is based on inclusion of a bulky hydrophobic part of the molecules, preferably aromatic moieties, in the hydrophobic cavity of the CD. An additional requirement is that secondary interactions have to take effect: these include dipoledipole interactions or hydrogen bonds between the hydroxyl groups at position 2 or 3 at the mouth of the CD and polar substituents close to the chiral center of the analyte. Since uncharged CDs migrate at the same velocity as the electroosmotic flow (EOF), they only allow the separation of charged analytes. The chiral resolution of 
uncharged analytes can be performed by micellar electrokinetic chromatography (MEKC) or by using charged CD derivatives.

CE is a convenient technique for the study of binding constants in real separation systems without any adaptations and approximations. Thus, this technique allows the measurement of binding constants under the exact conditions at which enantioseparations are performed. This constitutes its main advantage versus other separation techniques. In addition, the small sample and buffer amounts needed in CE together with its versatility enable the study of different possibilities of analyte-selector bindings $[13,14]$.

Enantiomeric separation of the structural analogs uniconazole and diniconazole by CD-modified MEKC (CDMEKC) by using as separation solution $100 \mathrm{~mm}$ sodium dodecyl sulfate (SDS) and $2 \mathrm{~m}$ urea in $100 \mathrm{~mm}$ borate buffer, pH 9.0, containing $50 \mathrm{~mm} \gamma-\mathrm{CD}$ and 5\% 2-methyl-2propanol, has been reported [1, 2]. Under these conditions, enantioresolution of fungicides was achieved in $\mathbf{4 0}$ min. The aim of this work was the selection of the optimal experimental conditions to enable fast enantiomeric separation of the fungicides uniconazole and diniconazole. A systematic evaluation of several chiral selectors, and a study of the influence of other experimental conditions, such as chiral selector concentration and temperature, on the enantiomeric separation were made. The usefulness of anionic $C D$ derivatives in the separation of fungicides was evaluated.

\section{Materials and methods}

\subsection{Chemicals and samples}

All reagents were of analytical grade. Taurocholic acid sodium salt (STC) and cholic acid sodium salt (SC) were purchased from Sigma (St. Louis, MO, USA); sodium dihydrogen phosphate, tris(hydroxymethyl)aminomethane hydrochloride (Tris), 2-( $\mathrm{N}$-morpholino) ethanesulfonic acid (MES), sodium dodecyl sulfate (SDS), and sodium hydroxide were from Merck (Darmstadt, Germany); ammonium formate, ammonium acetate and $\beta-C D$ were from Fluka (Buchs, Switzerland); (2-hydroxy)propyl $\beta$-CD (HP$\beta-C D$; with a substitution degree, s.d., of approximately 3 ), carboxymethylated- $\beta$-cyclodextrin (CM- $\beta-C D$, s.d. 3), carboxymethylated- $\gamma$-cyclodextrin (CM- $\gamma$-CD, s.d. 3 ), succinylated $\beta$-cyclodextrin (Succ- $\beta-C D$, s.d. $~ 3.5$ ) and succinylated $\gamma$-cyclodextrin (Succ- $\gamma$-CD, s.d. $\sim 3$ ) were obtained from Cyclolab (Budapest, Hungary). Water used to prepare solutions was purified through a Milli-Q system from Millipore (Bedford, MA, USA). All solutions were filtered through $45 \mu \mathrm{m}$ pore size disposable nylon filters from Scientific Resources (Eatontown, NJ, USA). The fungicides uniconazole (beta-((4-chlorophenyl)methylene)-alpha-(1,1-dimethylethyl)-1H-1,2,4-triazole-1-ethanol) and diniconazole (beta-((2,4-dichlorophenyl)methylene)-alpha-(1,1-dimethylethyl)-1H-1,2,4-triazole-1-ethanol) were purchased from ChemService (West Chester, PA, USA) and Dr. Ehrenstorfer Reference Materials (Augsburg, Germany), respectively. Figure 1 shows the structures of the fungicides uniconazole and diniconazole $[1,2]$. Methanol of HPLC grade (Labscan, Dublin, Ireland) was used for the preparation of fungicide solutions.

\subsection{Apparatus}

Two CE instruments were used: (i) a Prince model from LauerLabs (Emmen, The Netherlands), equipped with a Lambda 1000 UV detector and an acquisition data system Model Star 4.5 from Varian Associates (Sugar Land, TX, USA); and (ii) an HP ${ }^{3 D}$ CE system (Hewlett-Packard, Waldbronn, Germany) equipped with an on-column diode array detector (DAD) and HP 3D-CE Chemstation software. A $50 \mu \mathrm{m}$ inner diameter (ID) and $375 \mu \mathrm{m}$ outer diameter (OD) fused-silica capillary with an effective length of $50 \mathrm{~cm}(65 \mathrm{~cm}$ total length for the LauerLabs instrument and $58.5 \mathrm{~cm}$ for the $\mathrm{HP}^{3 \mathrm{D}}$ system) was employed (Polymicro Technologies, Phoenix, AZ, USA). Injection was performed hydrodynamically at 50 or 30 mbar for $2 \mathrm{~s}$. Usual CE conditions for separations of the fungicides uniconazole and diniconazole were: applied voltage, $20 \mathrm{kV}$; detection wavelength, $220 \mathrm{~nm}$; and capillary temperature set at $20,30,40$ or $50^{\circ} \mathrm{C}$. Electrolytic solutions were degassed in an ultrasonic cleaner KM from Raypa (Barcelona, Spain). A $654 \mathrm{pH}$ meter from Metrohm (Herisau, Switzerland) was employed to adjust the $\mathrm{pH}$ of the separation buffers.

\subsection{Procedure}

Buffers were prepared by dissolving the appropriate amount of sodium dihydrogen phosphate, ammonium formate, ammonium acetate, Tris or MES in Milli-Q water,

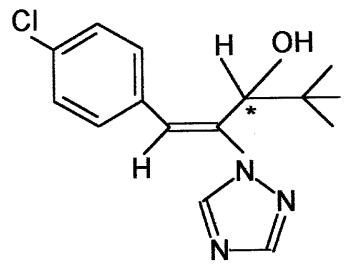

Uniconazole<smiles>CC(C)(C)[C@H](O)/C(=C/c1ccc(Cl)cc1Cl)n1cncn1</smiles>

Diniconazole
Figure 1. Structures of the fungicides uniconazole and diniconazole. 
and adjusting the $\mathrm{pH}$ to the desired value with a $1 \mathrm{~m}$ solution of sodium hydroxide (for phosphate, Tris, or MES buffers), formic acid (for formate buffer) or acetic acid (for acetate buffer). In order to obtain the electrolytic solutions for the enantiomeric separations, the chiral selector (bile salt or negatively charged CDs alone or mixed with the neutral HP- $\beta-C D$ ) was dissolved in the corresponding buffer solution. Finally, after adding additives such as $\mathrm{SDS}$, the $\mathrm{pH}$ was adjusted to the desired value. When methanol was used as additive, it was added after electroIytic solution preparation. $5 \mathrm{mg} / \mathrm{mL}$ stock solutions of uniconazole and diniconazole were prepared by dissolving $10 \mathrm{mg}$ of compound in $2 \mathrm{~mL}$ of methanol. The working solutions were obtained at $0.2 \mathrm{mg} / \mathrm{mL}$ by dilution of the stock solutions in methanol. In order to obtain good peak shapes and reproducible retention data, the capillary was conditioned at the beginning of the day, prior to each injection and at the end of the day. In all cases, the conditioning run included the following steps: (i) 2-min rinse with Milli-Q water, (ii) 2-min rinse with $0.1 \mathrm{M}$ sodium hydroxide, and (iii) 2-min rinse with Milli-Q water at 1000 mbar. Before sample injection the capillary was also rinsed with the running buffer for $2 \mathrm{~min}$.

\subsection{Data treatment}

Enantiomeric resolution was calculated by the following expression:

$R_{\mathrm{s}}=1.18\left(t_{2}-t_{1}\right) /\left(w_{1}+w_{2}\right)$

where $t_{1}$ and $t_{2}$ are the two enantiomers migration times and $w_{1}, w_{2}$ are the middle high widths of the corresponding peaks. The inclusion complexes binding constants for each enantiomer-CM- $\gamma$-CD pair were calculated, from electrophoretic mobilities, using linear plotting approaches by least-squares methods. From the experimental data, the electrophoretic mobility of each enantiomer $\left(\mu_{\mathrm{i}}\right)$ was determined by the following equation:

$\mu_{\mathrm{i}}=\frac{\mathrm{IL}}{V}\left(\frac{1}{t_{\mathrm{i}}}-\frac{1}{t_{0}}\right)$

where $L$ and $I$ are the total capillary length and the length to the detector, respectively, $V$ is the run voltage, $t_{\mathrm{i}}$ is the enantiomer migration time, and $t_{\mathrm{o}}$ is the migration time of methanol, used as neutral marker to correct changes in solution viscosity caused by variations in CD concentration. Experimental data were manipulated and parameters were calculated using Excel 7.0 from Microsoft Office software. The uncertainties in the binding constants, mobilities, and thermodynamic parameters listed in
Tables 3-5 were calculated by error propagation methods using the errors in the slopes and intercepts obtained by least-squares methods [15].

\section{Results and discussion}

\subsection{Enantiomeric separation of uniconazole and diniconazole}

In preliminary experiments, the enantiomeric resolution of fungicides was investigated by using different chiral selectors in the separation buffer. Charged CD derivatives were employed in EKC; and bile salts and mixtures of SDS and neutral and charged CDs were used in micellar EKC (MEKC). The use of anionic CD derivatives provided satisfactory results. The derivatives Succ- $\beta-C D$, Succ- $\gamma-C D$, CM- $\beta-C D$, and $C M-\gamma-C D$, and the mixtures $\mathrm{HP}-\beta-\mathrm{CD} / \mathrm{CM}-\gamma-\mathrm{CD}$ were evaluated with different buffers and $\mathrm{pH}$ values (experimental conditions are summarized in Table 1). The use of Succ- $\beta-C D$ and Succ- $\gamma-C D$ alone as chiral selectors in the separation buffer did not produce the enantioseparation of any of the fungicides. However, when $\mathrm{CM}-\beta-\mathrm{CD}$ and $\mathrm{CM}-\gamma-\mathrm{CD}$ were employed as chiral selectors, the chiral recognition of uniconazole and diniconazole was achieved. As can be seen in Table 1, the best resolutions were achieved when the derivative $\mathrm{CM}-\gamma-\mathrm{CD}$ in $50 \mathrm{~mm}$ phosphate buffer at $\mathrm{pH} 6.5$ was used. The use of a lower $\mathrm{pH}$ (formate buffer, $\mathrm{pH} 4$ ) improved the uniconazole resolution, but a decrease in the diniconazole resolution and an increase in the migration times were also produced.

Since mixtures of CDs may originate changes in selectivity, mixtures of $\mathrm{HP}-\beta-\mathrm{CD}$ and $\mathrm{CM}-\gamma-\mathrm{CD}$ were tested. Under these conditions, the resolutions obtained were worse than those achieved when CM- $\gamma$-CD was used as unique chiral selector. In this case, the loss in resolution could be caused by both a competitive effect between both CDs and the increase in the EOF. The use of methanol as additive in the CD mixtures (50 $\mathrm{mm}$ formate buffer, $\mathrm{pH}$ 6.5) gave rise to a slight decrease in the uniconazole resolution, while an improvement in the diniconazole resolution was achieved with respect to that obtained in the same buffer without methanol. A slight increase in the migration times was also produced in the presence of methanol.

Uniconazole and diniconazole were also injected in several MEKC systems in which different buffers, $50 \mathrm{~mm}$ in SDS and $10 \mathrm{~mm}$ in HP- $\beta-C D, \beta-C D$ or Succ- $\beta-C D$ were used. Under all these conditions, no chiral recognition was observed for the fungicides studied. When two bile salts, SC and STC were used as chiral selectors (two concentrations tested in $50 \mathrm{~mm}$ phosphate buffer at 
Table 1. Some experimental conditions tested for the enantiomeric separation of uniconazole and diniconazole

\begin{tabular}{|c|c|c|c|c|c|c|c|}
\hline Chiral & Chiral selector & BGE & Additives & & nazole & Dinico & azole \\
\hline selector & concentration & & & & $R_{\mathrm{s}}^{\mathrm{c})}$ & $t^{\mathrm{b})}$ & $R_{\mathrm{s}}{ }^{\mathrm{c})}$ \\
\hline$C M-\beta-C D$ & $10 \mathrm{~mm}$ & 50 mм phosphate/pH 6.5 & - & 5.77 & 0.52 & 5.70 & - \\
\hline & $20 \mathrm{~mm}$ & 50 mм phosphate/pH 6.5 & - & 6.73 & - & 6.75 & - \\
\hline & $10 \mathrm{~mm}$ & $50 \mathrm{~mm}$ acetate/pH 4.5 & - & 11.19 & 0.89 & 10.67 & 0.77 \\
\hline CM- $\gamma-C D$ & $5 \mathrm{~mm}$ & 100 mм phosphate/pH 6.5 & - & 3.90 & 0.63 & 4.04 & 0.62 \\
\hline & $10 \mathrm{~mm}$ & 100 mм phosphate/pH 6.5 & - & 4.14 & 1.05 & 4.59 & 1.05 \\
\hline & $5 \mathrm{~mm}$ & 50 mм phosphate/pH 6.5 & - & 4.72 & 1.09 & 4.93 & 0.82 \\
\hline & $10 \mathrm{~mm}$ & 50 mм phosphate/pH 6.5 & - & 4.98 & 1.41 & 5.20 & 1.06 \\
\hline & $15 \mathrm{~mm}$ & 50 mм phosphate/pH 6.5 & - & 5.76 & 2.26 & 6.17 & 1.61 \\
\hline & $20 \mathrm{~mm}$ & 50 mм phosphate/pH 6.5 & - & 6.71 & 1.92 & 7.54 & 1.77 \\
\hline & $15 \mathrm{~mm}$ & $50 \mathrm{~mm}$ formate/pH 4 & - & 12.96 & 3 & 15.45 & 1.2 \\
\hline 10 mм HP- $\beta-C D$ & & 50 mм phosphate/pH 6.5 & - & 4.00 & 0.51 & 4.19 & 0.65 \\
\hline$+10 \mathrm{~mm} C M-\gamma-C D$ & & 50 mм MES/pH 6.5 & - & 4.56 & 0.58 & 4.74 & 0.98 \\
\hline & & $50 \mathrm{~mm}$ formate/pH 5 & - & 6.92 & 1.46 & 7.31 & 1.27 \\
\hline & & $50 \mathrm{~mm}$ formate $/ \mathrm{pH} 6.5$ & - & 4.34 & 0.66 & 4.09 & 0.44 \\
\hline & & $50 \mathrm{~mm}$ formate/pH 6.5 & $5 \%$ methanol & 4.79 & 0.51 & 4.89 & 0.77 \\
\hline & & $50 \mathrm{~mm}$ formate/pH 6.5 & $10 \%$ methanol & 5.92 & 0.53 & 6.15 & 1.20 \\
\hline Succ- $\beta-C D$ & $10 \mathrm{~mm}$ & 50 mм phosphate/pH 6.5 & - & 7.82 & - & 7.68 & - \\
\hline & $20 \mathrm{~mm}$ & 50 mм phosphate/pH 6.5 & - & 15.19 & - & 16.23 & - \\
\hline & $10 \mathrm{~mm}$ & $50 \mathrm{~mm}$ MES/pH 6.5 & - & 5.97 & - & 5.87 & - \\
\hline & $10 \mathrm{~mm}$ & 50 mм phosphate/pH 6.5 & $50 \mathrm{~mm}$ SDS & - & - & 11.34 & - \\
\hline & $10 \mathrm{~mm}$ & 50 mм phosphate/pH 6.5 & $2 \%$ methanol & 8.04 & - & 8.03 & - \\
\hline & $10 \mathrm{~mm}$ & $50 \mathrm{~mm}$ phosphate/pH 6.5 & $5 \%$ methanol & 9.65 & - & 9.49 & - \\
\hline Succ- $\gamma-C D$ & $10 \mathrm{~mm}$ & 50 mм phosphate/pH 6.5 & - & 4.92 & - & 4.83 & - \\
\hline
\end{tabular}

a) Averaged migration time for uniconazole enantiomers, in min

b) Averaged migration time for diniconazole enantiomers, in min

c) Resolution between enantiomers

In all cases CE conditions were: injection, 50 mbar for $2 \mathrm{~s}$; run voltage, $20 \mathrm{kV}$; temperature, $40^{\circ} \mathrm{C}$; detection wavelength, $220 \mathrm{~nm}$.

$\mathrm{pH}$ 6.5), no chiral recognition was observed. Among the different chiral selectors considered, CM- $\gamma$-CD was found to be the most appropriate for the uniconazole and diniconazole enantioseparation. Next, a more exhaustive study on the influence of several parameters on the enantiomeric resolution, using $\mathrm{CM}-\gamma-\mathrm{CD}$ as chiral selector, was performed in order to improve the resolution of the compounds studied.

\subsection{Influence of the chiral selector concentration and temperature on the enantiomeric separation of uniconazole and diniconazole with $\mathrm{CM}-\gamma-\mathrm{CD}$}

A solution containing $50 \mathrm{~mm}$ phosphate buffer at $\mathrm{pH} 6.5$ with $C M-\gamma-C D$ as chiral selector was chosen to study the influence of $C D$ concentration and temperature on the enantiomeric resolution of uniconazole and diniconazole. The concentration of CM- $\gamma-\mathrm{CD}$ was varied in the range of 2-20 mm. Chiral recognition of uniconazole and dinicona- zole was achieved for all concentrations and temperatures tested. In addition, several of these conditions enabled the fast separation of mixtures of the two fungicides. Table 2 shows the averaged migration times obtained for uniconazole and diniconazole enantiomers, and the resolution between the second-migrating enantiomer of uniconazole and the first-migrating enantiomer of diniconazole at different temperatures and $\mathrm{CM}-\gamma-\mathrm{CD}$ concentrations under experimental conditions, enabling the enantioseparation of the two fungicides in mixtures. It can be observed that averaged migration times decreased when the CM- $\gamma$-CD concentration was decreased at a constant temperature, according to a decrease in the viscosity of the solution. In addition, for a constant CM- $\gamma-C D$ concentration, averaged migration times decreased with increasing temperature due to the decrease obtained for the viscosity at increasing temperature values. Table 2 also shows that low $\mathrm{CM}-\gamma-\mathrm{CD}$ concentrations together with a temperature of $50^{\circ} \mathrm{C}$ are the most useful experimental conditions at which to perform the enantiomeric separa- 
tion of mixtures of uniconazole and diniconazole. Although a $2 \mathrm{~mm}$ concentration of $\mathrm{CM}-\gamma-\mathrm{CD}$ at $50^{\circ} \mathrm{C}$ enabled the individual baseline resolution of the two enan-

Table 2. $C M-\gamma-C D$ concentration and temperature conditions enabling the enantioseparation of uniconazole and diniconazole mixtures

\begin{tabular}{ccccc}
\hline $\begin{array}{c}\text { [CM- } \gamma-\mathrm{CD}] \\
(\mathrm{mM})\end{array}$ & $T\left({ }^{\circ} \mathrm{C}\right)$ & $t^{\mathrm{a})}$ & $t^{\mathrm{b})}$ & $R_{\mathrm{s}}^{\mathrm{c})}$ \\
\hline 2 & 20 & 6.87 & 7.14 & 2.00 \\
5 & 20 & 8.40 & 8.95 & 1.74 \\
2 & 30 & 5.81 & 6.15 & 1.58 \\
5 & 30 & 6.91 & 7.28 & 1.74 \\
2 & 40 & 4.95 & 5.19 & 1.85 \\
5 & 40 & 5.74 & 6.05 & 1.85 \\
2 & 50 & 4.36 & 4.52 & 1.58 \\
5 & 50 & 5.04 & 5.10 & 1.88 \\
10 & 50 & 5.35 & 5.62 & 1.75
\end{tabular}

a) Averaged migration time for uniconazole enantiomers, in $\min$

b) Averaged migration time for diniconazole enantiomers, in min

c) Resolution between the second-migration enantiomer of uniconazole and the first-migrating enantiomer of diniconazole.

BGE, solution containing $50 \mathrm{~mm}$ phosphate buffer at $\mathrm{pH}$ 6.5. In all cases $\mathrm{CE}$ conditions were: injection, $50 \mathrm{mbar}$ for $2 \mathrm{~s}$; run voltage, $20 \mathrm{kV}$; detection wavelength, $220 \mathrm{~nm}$

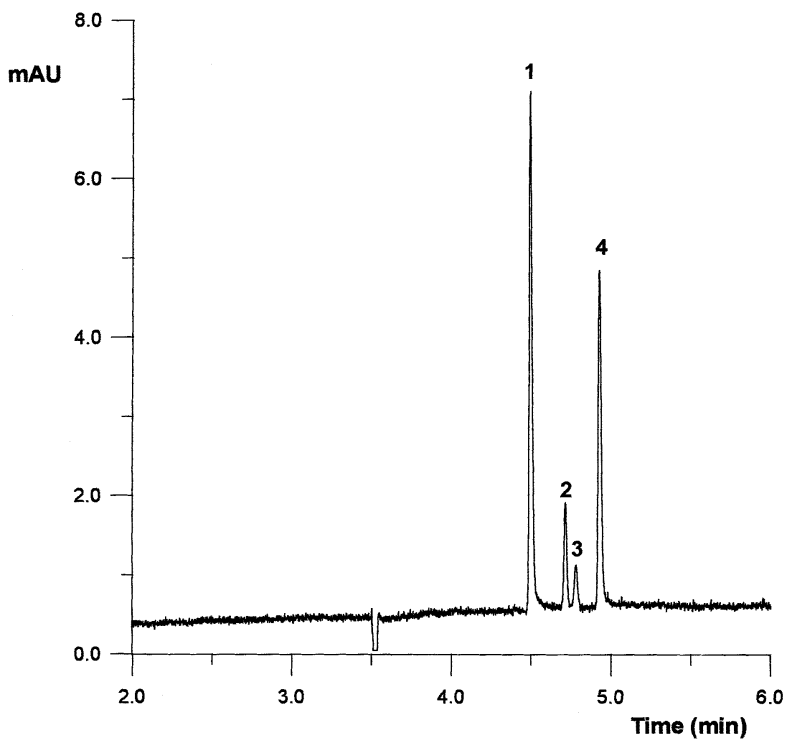

Figure 2. Enantiomeric separation of uniconazole (peaks 1 and 2) and diniconazole (peaks 3 and 4). BGE, $50 \mathrm{~mm}$ phosphate buffer at $\mathrm{pH} 6.5$ containing $5 \mathrm{~mm}$ CM- $\gamma$-CD. Capillary, $50 \mu \mathrm{m} \times 50 \mathrm{~cm}$ uncoated fused silica; injection, $50 \mathrm{mbar}$ for $2 \mathrm{~s}$; run voltage, $20 \mathrm{kV}$; temperature, $50^{\circ} \mathrm{C}$; detection wavelength, $220 \mathrm{~nm}$. tiomers of each fungicide, a concentration of $5 \mathrm{~mm}$ of $\mathrm{CM}$ $\gamma$-CD was chosen in order to achieve a good resolution between the second-migrating enantiomer of uniconazole and the first-migrating enantiomer of diniconazole in a mixture of these two fungicides. Figure 2 shows the separation of uniconazole and diniconazole performed at $50^{\circ} \mathrm{C}$, using an electrolytic solution containing $50 \mathrm{~mm}$ phosphate buffer at $\mathrm{pH} 6.5$ and $5 \mathrm{~mm}$ CM- $\gamma$-CD. These conditions allow the fast enantioseparation of a mixture of the two fungicides in about $5 \mathrm{~min}$.
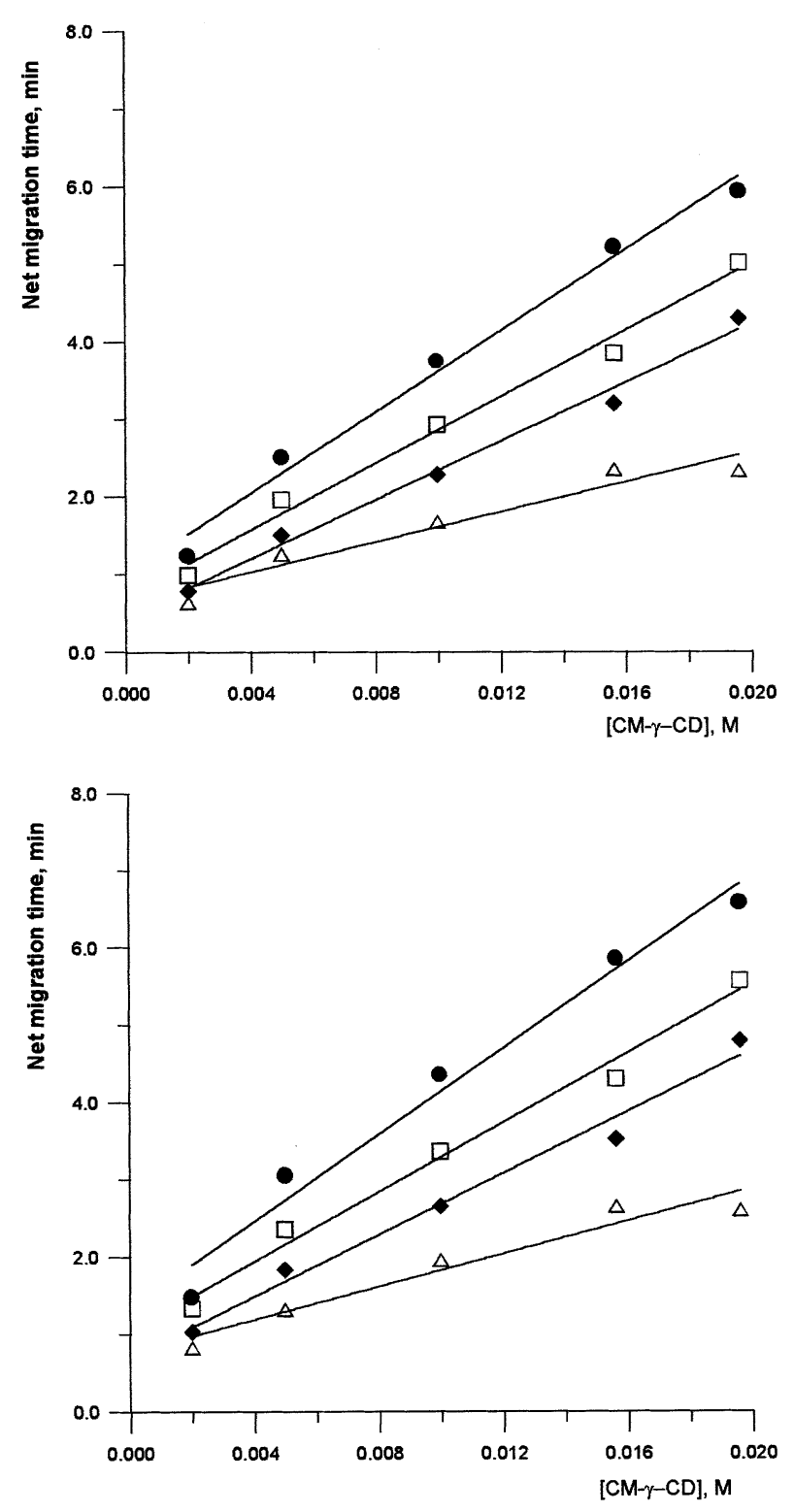

Figure 3. Dependence of net migration times of uniconazole (upper part) and diniconazole (lower part) upon the

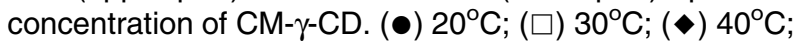
$(\triangle) 50^{\circ} \mathrm{C}$. 
From these results, the enantiomeric ratio could be determined for the two fungicides investigated. Thus, the enantiomer proportions were calculated from the corresponding areas in different electropherograms, obtaining values of about 80:20 (peak 1 : peak 2) for uniconazole and 20:80 (peak 3 : peak 4) for diniconazole. Finally, Fig. 3 shows plots for the net migration time of uniconazole (upper part) and diniconazole (lower part) at several temperatures as a function of the CM- $\gamma-\mathrm{CD}$ concentration. The net migration time was defined as $t_{i}^{\prime}-t_{0}$, where $t_{i}^{\prime}$ is the average of the migration times of both enantiomers, and $t_{\mathrm{o}}$ is the migration time of the neutral marker. It can be observed that net migration times increased with increasing $\mathrm{CM}-\gamma-\mathrm{CD}$ concentrations by means of a linear variation. This effect could be explained by the existence of a greater proportion of solute complexed under these conditions. In addition, slopes of the linear plots increased with decreasing temperature due to the longer net migration times obtained at lower temperatures for a constant concentration of $C M-\gamma-C D$ according to a decrease in the EOF caused by changes in the viscosity.

\subsection{Determination of apparent analyte-selector binding constants for enantiomers}

Knowledge of binding constants when inclusion complexation occurs is of interest in order to appreciate the extent of an analyte inclusion into the cavity of a CD. A theoretical model relating mobility to the concentraton of the $C D$ selector was developed by Wren and Rowe [16, 17]. Chi-
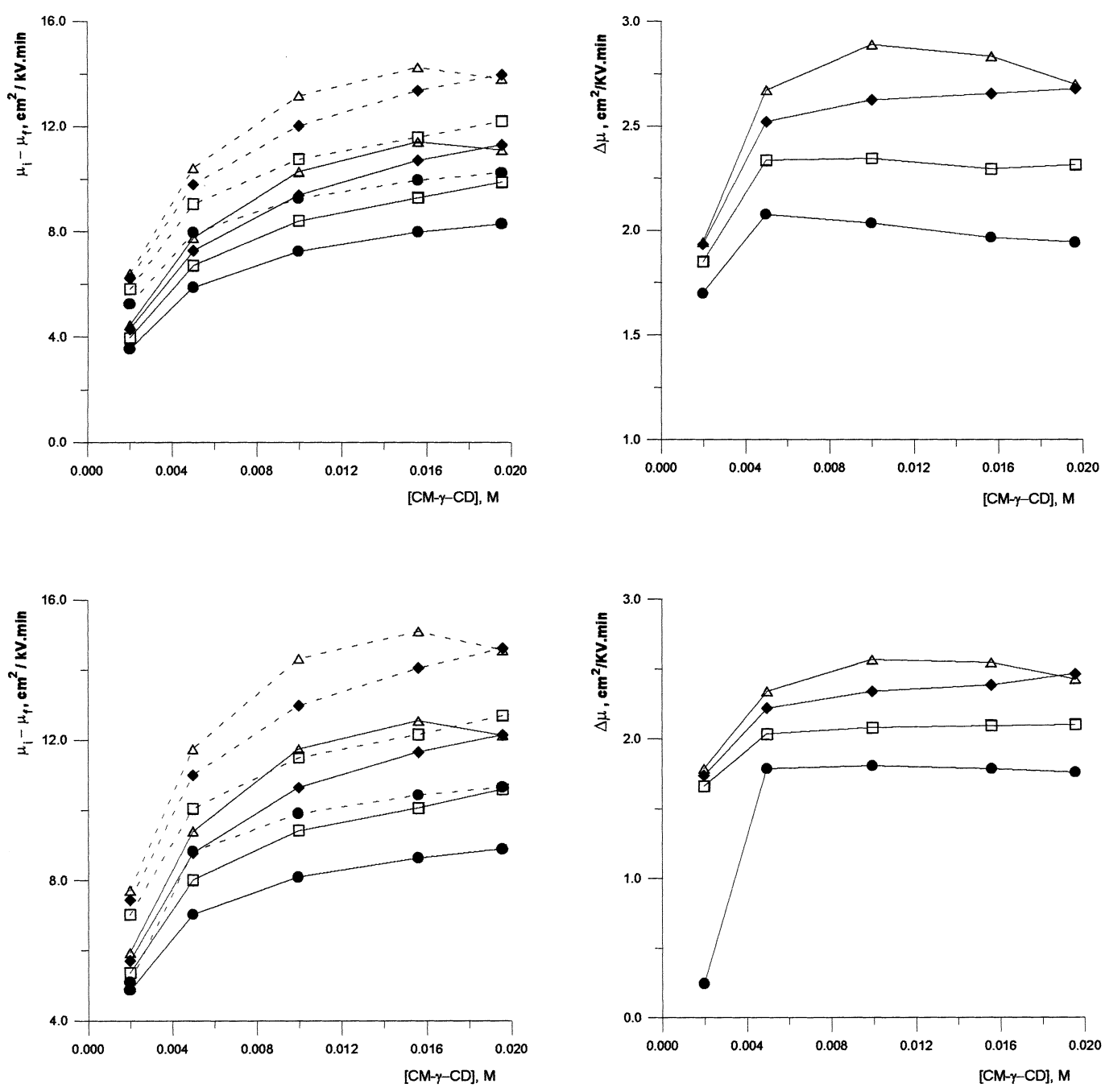

Figure 4. Plots of the change in solute mobility and differences in enantiomers electrophoretic mobilities as a function of $\mathrm{CM}-\gamma-\mathrm{CD}$ concentration for uniconazole (upper part) and diniconazole (lower part). The curves were drawn by hand to illustrate the general trends in the data. $(\bullet) 20^{\circ} \mathrm{C} ;(\square) 30^{\circ} \mathrm{C}$; (•) $40^{\circ} \mathrm{C} ;(\triangle) 50^{\circ} \mathrm{C}$. 
ral recognition will depend on the difference between the electrophoretic mobilities of each free and complexed enantiomer. Binding constants can be determined using the following expression:

$K[\mathrm{~L}]=\left(\frac{\mu_{\mathrm{f}}-\mu_{\mathrm{i}}}{\mu_{\mathrm{i}}-\mu_{\mathrm{c}}}\right)$

where $K$ is the binding constant, $[L]$ is the equilibrium concentration of uncomplexed ligand, $\mu_{\mathrm{f}}$ and $\mu_{\mathrm{c}}$ are the electrophoretic mobilities of the free and complexed solute, and $\mu_{\mathrm{i}}$ is the solute mobility at the ligand concentration [L].

The mobility of the complex, $\mu_{\mathrm{c}}$, must be measured in order to use Eq. (3) directly to estimate binding constants. Although it can be approached to the solute mobility at very high concentrations of $C D$, sometimes its measurement is difficult or impossible due to the difficulty of finding suitable CD markers and reaching saturating conditions. In addition, an increase in the chiral selector concentration leads to some changes in electrophoretic mobility (due to changes in buffer viscosity, EOF, etc.) which are not related to the selector-selectand binding [13]. This can be a source of systematic error in this technique. In order to avoid this problem, Eq. (3) can be rearranged in many plotting forms that do not require the direct meas-

$\frac{1}{\mu_{i}-\mu_{f}}=\frac{1}{\left(\mu_{c}-\mu_{f}\right) K} \frac{1}{[L]}+\frac{1}{\left(\mu_{c}-\mu_{f}\right)}$ urement of $\mu_{\mathrm{c}}$. The plotting forms of these equations, summarized by Rundlett and Armstrong [14], are the following:

Double reciprocal

$y$-reciprocal

$\frac{[\mathrm{L}]}{\mu_{\mathrm{i}}-\mu_{\mathrm{f}}}=\frac{1}{\mu_{\mathrm{c}}-\mu_{\mathrm{f}}}[\mathrm{L}]+\frac{1}{\left(\mu_{\mathrm{c}}-\mu_{\mathrm{f}}\right) K}$

$x$-reciprocal

$\frac{\mu_{\mathrm{i}}-\mu_{\mathrm{f}}}{[\mathrm{L}]}=-K\left(\mu_{\mathrm{i}}-\mu_{\mathrm{f}}\right)+K\left(\mu_{\mathrm{c}}-\mu_{\mathrm{f}}\right)$

To calculate the CD-analyte binding constants, the mobilities for enantiomers were measured at different concentrations of chiral selector. Plots showing the effect of CM$\gamma$-CD concentration on solute mobilities at several temperatures are shown in Fig. 4. At the four values of the temperature checked for uniconazole and diniconazole, electrophoretic mobilities can be seen to increase when increasing the CD concentration in all ranges tested. On the other hand, differences in electrophoretic mobilities of enantiomers, $\Delta \mu_{\mathrm{i}}$, initially increased with increasing CD concentrations, and remained more or less constant or decreased slightly after a maximum was reached. Furthermore, these differences were larger at higher temperatures.

Table 3. Apparent binding constants and enantioselectivities of complexation for uniconazole with $\mathrm{CM}-\gamma-\mathrm{CD}$ in $50 \mathrm{~mm}$ phosphate buffer ( $\mathrm{pH} 6.5)$ at several temperatures

\begin{tabular}{|c|c|c|c|c|c|c|c|}
\hline Plotting method & $\log K_{1}^{a)}$ & $\log K_{2}^{a)}$ & $\alpha^{b)}$ & $\mu_{c}(1)^{c)}$ & $\mu_{c}(2)^{c)}$ & $r^{2}(1)^{d)}$ & $r^{2}(2)^{d)}$ \\
\hline \multicolumn{8}{|l|}{$20^{\circ} \mathrm{C}$} \\
\hline Double reciprocal & $2.458 \pm 0.008$ & $2.629 \pm 0.009$ & 1.48 & $-9.83 \pm 0.13$ & $-11.52 \pm 0.12$ & 0.999 & 0.999 \\
\hline$y$-Reciprocal & $2.47 \pm 0.01$ & $2.64 \pm 0.01$ & 1.49 & $-9.72 \pm 0.06$ & $-11.42 \pm 0.06$ & 0.9999 & 0.9999 \\
\hline$x$-Reciprocal & $2.46 \pm 0.01$ & $2.63 \pm 0.01$ & 1.48 & $-9.8 \pm 0.3$ & $-11.5 \pm 0.5$ & 0.998 & 0.997 \\
\hline \multicolumn{8}{|l|}{$30^{\circ} \mathrm{C}$} \\
\hline Double reciprocal & $2.410 \pm 0.009$ & $2.568 \pm 0.009$ & 1.44 & $-11.8 \pm 0.2$ & $-13.8 \pm 0.2$ & 0.999 & 0.999 \\
\hline$y$-Reciprocal & $2.41 \pm 0.02$ & $2.57 \pm 0.03$ & 1.43 & $-11.7 \pm 0.2$ & $-13.8 \pm 0.2$ & 0.999 & 0.9995 \\
\hline$x$-Reciprocal & $2.415 \pm 0.014$ & $2.571 \pm 0.015$ & 1.43 & $-11.7 \pm 0.5$ & $-13.7 \pm 0.6$ & 0.997 & 0.997 \\
\hline \multicolumn{8}{|l|}{$40^{\circ} \mathrm{C}$} \\
\hline Double reciprocal & $2.364 \pm 0.005$ & $2.506 \pm 0.006$ & 1.39 & $-13.64 \pm 0.12$ & $-16.00 \pm 0.15$ & 0.9998 & 0.9996 \\
\hline$y$-Reciprocal & $2.346 \pm 0.013$ & $2.48 \pm 0.02$ & 1.37 & $-13.84 \pm 0.14$ & $-16.2 \pm 0.2$ & 0.9997 & 0.9997 \\
\hline$x$-Reciprocal & $2.36 \pm 0.01$ & $2.50 \pm 0.01$ & 1.39 & $-13.7 \pm 0.4$ & $-16.1 \pm 0.5$ & 0.999 & 0.998 \\
\hline \multicolumn{8}{|l|}{$50^{\circ} \mathrm{C}$} \\
\hline Double reciprocal & $2.357 \pm 0.005$ & $2.461 \pm 0.007$ & 1.27 & $-15.00 \pm 0.15$ & $-17.6 \pm 0.2$ & 0.9999 & 0.9998 \\
\hline$y$-Reciprocal & $2.344 \pm 0.013$ & $2.48 \pm 0.02$ & 1.36 & $-14.8 \pm 0.2$ & $-17.4 \pm 0.2$ & 0.9997 & 0.9997 \\
\hline$x$-Reciprocal & $2.334 \pm 0.011$ & $2.464 \pm 0.012$ & 1.35 & $-14.9 \pm 0.4$ & $-17.6 \pm 0.2$ & 0.999 & 0.999 \\
\hline
\end{tabular}

a) Binding constants $\left(\mathrm{M}^{-1}\right)$ for the first- and second-migrating enantiomers

b) Enantioselectivities of complexation ( $\alpha$ ) were calculated as $\alpha=K_{2} / K_{1}$

c) Electrophoretic mobilities of the enantiomer-CD complex in $\mathrm{cm}^{2} \mathrm{kV}^{-1} \mathrm{~min}^{-1}$ for the first- and second-migrating enantiomers

d) Squared correlation coefficient obtained by the least-squares method 
Table 4. Apparent binding constants and enantioselectivities of complexation for diniconazole with $\mathrm{CM}-\gamma-\mathrm{CD}$ in $50 \mathrm{~mm}$ phosphate buffer ( $\mathrm{pH} 6.5)$ at several temperatures ${ }^{\mathrm{a})}$

\begin{tabular}{|c|c|c|c|c|c|c|c|}
\hline Plotting method & $\log K_{1}$ & $\log K_{2}$ & $\alpha$ & $\mu_{\mathrm{c}}(1)$ & $\mu_{\mathrm{c}}(2)$ & $r^{2}(1)$ & $r^{2}(2)$ \\
\hline \multicolumn{8}{|l|}{$20^{\circ} \mathrm{C}$} \\
\hline Double reciprocal & $2.700 \pm 0.006$ & $2.83 \pm 0.01$ & 1.36 & $-9.78 \pm 0.06$ & $-11.42 \pm 0.05$ & 0.9996 & 0.999 \\
\hline$y$-Reciprocal & $2.700 \pm 0.013$ & $2.82 \pm 0.02$ & 1.31 & $-9.78 \pm 0.05$ & $-11.47 \pm 0.05$ & 0.9999 & 1.0 \\
\hline$x$-Reciprocal & $2.701 \pm 0.008$ & $2.831 \pm 0.014$ & 1.35 & $-9.8 \pm 0.2$ & $-11.4 \pm 0.5$ & 0.999 & 0.998 \\
\hline \multicolumn{8}{|l|}{$30^{\circ} \mathrm{C}$} \\
\hline Double reciprocal & $2.631 \pm 0.008$ & $2.721 \pm 0.008$ & 1.23 & $-11.71 \pm 0.10$ & $-13.78 \pm 0.11$ & 0.999 & 0.999 \\
\hline$y$-Reciprocal & $2.62 \pm 0.03$ & $2.71 \pm 0.03$ & 1.23 & $-11.8 \pm 0.2$ & $-13.8 \pm 0.2$ & 0.999 & 0.9996 \\
\hline$x$-Reciprocal & $2.631 \pm 0.014$ & $2.721 \pm 0.014$ & 1.23 & $-11.7 \pm 0.5$ & $-13.8 \pm 0.6$ & 0.997 & 0.997 \\
\hline \multicolumn{8}{|l|}{$40^{\circ} \mathrm{C}$} \\
\hline Double reciprocal & $2.555 \pm 0.005$ & $2.635 \pm 0.006$ & 1.20 & $-13.74 \pm 0.08$ & $-16.15 \pm 0.11$ & 0.9998 & 0.9996 \\
\hline$y$-Reciprocal & $2.540 \pm 0.014$ & $2.61 \pm 0.02$ & 1.19 & $-13.87 \pm 0.10$ & $-16.32 \pm 0.14$ & 0.9998 & 0.9998 \\
\hline$x$-Reciprocal & $2.552 \pm 0.008$ & $2.63 \pm 0.01$ & 1.20 & $-13.8 \pm 0.3$ & $-16.2 \pm 0.5$ & 0.999 & 0.998 \\
\hline \multicolumn{8}{|l|}{$50^{\circ} \mathrm{C}$} \\
\hline Double reciprocal & $2.509 \pm 0.008$ & $2.588 \pm 0.009$ & 1.20 & $-15.2 \pm 0.2$ & $-17.8 \pm 0.2$ & 0.9996 & 0.999 \\
\hline$y$-Reciprocal & $2.53 \pm 0.02$ & $2.61 \pm 0.03$ & 1.20 & $-15.0 \pm 0.2$ & $-17.6 \pm 0.2$ & 0.9995 & 0.9995 \\
\hline$x$-Reciprocal & $2.512 \pm 0.015$ & $2.590 \pm 0.016$ & 1.20 & $-15.2 \pm 0.6$ & $-17.8 \pm 0.8$ & 0.998 & 0.997 \\
\hline
\end{tabular}

a) See Table 3 for abbreviations

Tables 3 and 4 list the apparent binding constants, enantioselectivities of complexation, and electrophoretic mobilities of the complexed solute, $\mu_{\mathrm{c}}$, calculated using Eqs. (4)-(6) for the chiral compounds studied and CM- $\gamma-C D$. The enantioselectivities of complexation $(\alpha)$ [13] were calculated as $\alpha=K_{2} / K_{1}$ where $K_{1}$ and $K_{2}$ are the binding constants for the first- and second-migrating enantiomers with $\mathrm{CM}-\gamma-\mathrm{CD}$. In all cases, reasonable results with acceptable error limits and good correlation coefficients were achieved; however, the correlation coefficients obtained by double and $y$-reciprocal methods were, in general, slightly higher than those for the $x$-reciprocal method.

As uniconazole and diniconazole are structurally similar, they also have similar values for the apparent binding constants, these constants being slightly lower for uniconazole. In all cases, good enantioselectivities of complexation were achieved ( $\alpha \geq 1$.19). On the other hand, both binding constants and enantioselectivities were slightly lower with increasing temperature. Finally, electrophoretic mobilities of the complexed solute, $\mu_{\mathrm{c}}$, increased with increasing temperature, in agreement with the increase in the EOF caused by changes in the viscosity.

Temperature dependence of the binding constants can be described as:

$$
\ln K=-\frac{\Delta H^{\circ}}{R T}+\frac{\Delta S^{\circ}}{R}
$$

where $\Delta H^{\circ}$ is the enthalpy change associated with inclusion complex formation, $\Delta S^{\circ}$ is the corresponding entropy change, and $R$ is the gas constant. As shown by Guillaume and Peyrin [18], thermodynamic data can give information about the solute-CD complexation mechanisms. Average logarithms of apparent binding constants determined from values calculated by different plotting methods at several temperatures were plotted against the reciprocal temperature, according to Eq. (7), to give straight lines. There were no available thermodynamic data in the literature concerning inclusion complex formation for these fungicides with CM- $\gamma-\mathrm{CD}$. The values of $\Delta H^{\mathrm{P}}$ and $\Delta S^{\circ}$ calculated from the above plots are listed in Table 5 for uniconazole and diniconazole enantiomers along with correlation coefficients for the plots and Gibbs

Table 5. Thermodynamic parameters for binding between uniconazole and diniconazole enantiomers and CM- $\gamma-C D$ in $50 \mathrm{~mm}$ phosphate buffer at $\mathrm{pH} 6.5$

\begin{tabular}{|c|c|c|c|c|}
\hline Analyte & $\begin{array}{c}\Delta H^{\circ} \\
\left(\mathrm{kJ} \mathrm{mol}^{-1}\right)\end{array}$ & $\begin{array}{c}\Delta S^{\circ} \\
\left.\mathrm{J} \mathrm{mol}^{-1} \mathrm{~K}^{-1}\right)\end{array}$ & $r^{a)}$ & $\begin{array}{c}\Delta G^{\circ}{ }_{298} \\
\left(\mathrm{~kJ} \mathrm{~mol}^{-1}\right)\end{array}$ \\
\hline Uniconazole $\left.{ }^{b}\right)$ & $-7.7 \pm 1.2$ & $21 \pm 4$ & 0.98 & -14 \\
\hline Uniconazole $^{\mathrm{c})}$ & $-10.5 \pm 1.1$ & $15 \pm 3$ & 0.99 & $-14.8 \pm 1.4$ \\
\hline Diniconazole $\left.^{b}\right)$ & $-11.4 \pm 1.2$ & $13 \pm 4$ & 0.99 & $-15 \pm 2$ \\
\hline Diniconazole & $-14 \pm 2$ & $5 \pm 7$ & 0.98 & -16 \\
\hline
\end{tabular}

a) Correlation coefficient obtained by the least-squares method

b) First-migrating enantiomer

c) Second-migrating enantiomer 
free energies $\Delta G^{\circ}$ for inclusion complex formation at $25^{\circ} \mathrm{C}$. Thus higher entropy $\left(\Delta S^{\circ}\right)$ and lesser enthalpy $\left(\Delta H^{\circ}\right)$ can be observed for the CM- $\gamma-\mathrm{CD}$ complex with uniconazole enantiomers than for diniconazole enantiomers. On the other hand, the inclusion into the cavity of the CM$\gamma-C D$ is favored for diniconazole compared with the uniconazole (as $\Delta G^{\circ}{ }_{298}$ values show) in agreement with a major migration time for diniconazole enantiomers.

\section{Concluding remarks}

The pairs of enantiomers of both uniconazole and diniconazole have been successfully separated by EKC employing $C M-\gamma-C D$ as a chiral selector. The use of an electrolytic solution containing $50 \mathrm{~mm}$ phosphate buffer at $\mathrm{pH} 6.5$ and $5 \mathrm{~mm} \mathrm{CM}-\gamma-\mathrm{CD}$ at $50^{\circ} \mathrm{C}$ allows the fast enantioseparation of uniconazole and diniconazole mixtures in about $5 \mathrm{~min}$. These results notably improve those reported in the literature describing the enantioseparation of these fungicides by CD-MEKC in $40 \mathrm{~min}$. On the other hand, the high efficiency of CE combined with plotting methods makes the estimation of apparent binding constants for enantiomers of uniconazole and diniconazole with $\mathrm{CM}-\gamma-\mathrm{CD}$ a simple, straightforward process. Binding constants for both compounds are similar, being slightly lower for uniconazole. Good enantioselectivities of complexation were achieved $(\alpha \geq 1.19)$ in all cases. The estimation of binding constants at several temperatures has enabled the determination of thermodynamic parameters related to the enantiomer $\mathrm{CM}-\gamma-\mathrm{CD}$ complexation.

The authors thank the Comunidad Autónoma de Madrid (Spain) for project 07M/0049/1998.

Received April 18, 2000

\section{References}

[1] Furuta, R., Doi, T., J. Chromatogr. A 1994, 676, 431-436.

[2] Furuta, R., Doi, T., Electrophoresis 1994, 15, 1322-1325.

[3] Ward, T. J., Anal. Chem. 1994, 66, 633A-640A.

[4] Lurie, I. S., J. Chromatogr. A 1997, 792, 297-307.

[5] Nishi, H., J. Chromatogr. A 1996, 735, 57-76.

[6] Vespalec, R., Boček, P., Electrophoresis 1997, 18, 843-852.

[7] Nishi, H., Terabe, S., J. Chromatogr. A 1995, 694, 245-276.

[8] Marina, M. L., Crego, A. L., J. Liq. Chromatogr. Rel. Technol. 1997, 20, 1337-1365.

[9] Gübitz, G., Schmid, M. G., J. Chromatogr. A 1997, 792, 179-225.

[10] Shamsi, S. A., Warner, I. M., Electrophoresis 1997, 18, 853-872.

[11] Lloyd, D. K., Aubry, A. F., Lorenzi, E. D., J. Chromatogr. A 1997, 792, 349-369.

[12] Armstrong, D. W., Nair, U. B., Electrophoresis 1997, 18, 2331-2342.

[13] Chankvetadze, B., Capillary Electrophoresis in Chiral Analysis, John Wiley \& Sons, Chichester 1997.

[14] Rundlett, K. L., Armstrong, D. W., J. Chromatogr. A 1996, 721, 173-186.

[15] Miller, J. C., Miller, J. N., Estadíistica para Química Analítica, Addison-Wesley Iberoamericana, Wilmington, DE, USA 1993.

[16] Wren, S. A. C., Rowe, R. C., J. Chromatogr. 1992, 609, 363-367.

[17] Wren, S. A. C., Rowe, R. C., J. Chromatogr. 1992, 603, 235-241.

[18] Guillaume, Y. C., Peyrin, E., Anal. Chem. 1999, 71, 2046-2052. 